\title{
Higher dietary and serum carotenoid levels are associated with lower carotid intima-media thickness in middle-aged and elderly people
}

\author{
Cheng Wang $^{1} \dagger$, Rui Qiu ${ }^{1} \dagger$, Yi Cao ${ }^{1}$, Wei-fu Ouyang ${ }^{2}$, Hua-bin $\mathrm{Li}^{1}$, Wen-hua Ling ${ }^{1}$ and Yu-Ming Chen ${ }^{1 *}$ \\ ${ }^{1}$ Guangdong Provincial Key Laboratory of Food, Nutrition and Health, School of Public Health, Sun Yat-sen University, \\ Guangzhou 510080, People's Republic of China \\ ${ }^{2}$ Laboratory Department, Guangdong General Hospital \& Guangdong Academy of Medical Sciences, Guangzhou 510080, \\ People's Republic of China
}

(Submitted 2 March 2017 - Final revision received 27 October 2017 - Accepted 1 November 2017)

\section{Abstract}

Several studies have suggested that higher carotenoid levels may be beneficial for atherosclerosis patients, but few studies have examined this relationship in the Chinese population. This cross-sectional study examined the association between the levels of carotenoids in diet and serum and carotid intima-media thickness (IMT) in Chinese adults aged 50-75 years in Guangzhou, China. Dietary intake was assessed using a FFQ. HPLC was used to assay the serum concentrations of $\alpha$-carotene, $\beta$-carotene, lutein + zeaxanthin, $\beta$-cryptoxanthin and lycopene. The IMT at the common carotid artery (CCA) and bifurcation of the carotid artery was measured by B-mode ultrasound. A total of 3707 and 2947 participants were included in the analyses of dietary and serum carotenoids. After adjustment for demographic, socio-economic and lifestyle factors, all the serum carotenoids levels except lycopene were found to be inversely associated with the IMT at the CCA and bifurcation $\left(P_{\text {trend }}<0 \cdot 001\right.$ to $\left.0 \cdot 013\right)$ in both men and women. The absolute mean differences in the IMT between the subjects in the extreme quartiles of serum carotenoid levels were $0.034 \mathrm{~mm}$ ( $\alpha$-carotene), $0.037 \mathrm{~mm}$ ( $\beta$-carotene), $0.032 \mathrm{~mm}$ (lutein + zeaxanthin), $0.030 \mathrm{~mm}$ ( $\beta$-cryptoxanthin), $0.015 \mathrm{~mm}($ lycopene) and $0.035 \mathrm{~mm}$ (total carotenoids) at the CCA; the corresponding values were 0.025, 0.053 0.043, 0.050, 0.011 and 0.042 mm at the bifurcation. The favourable associations were also observed between dietary carotenoids (except lycopene) and the CCA IMT. In conclusion, elevated carotenoid levels in diet and serum are associated with lower carotid IMT values (particular at the CCA) in Chinese adults.

Key words: Carotid intima-media thickness: Carotenoids: Serum: Atherosclerosis: Cross-sectional studies: Chinese population

CVD are the leading worldwide causes of mortality and morbidity $^{(1)}$. In China, nearly three million people died of CVD in 2012, accounting for $51 \%$ of all deaths ${ }^{(2)}$. The prevention of atherosclerosis is one of the major contemporary challenges in the primary prevention of $\mathrm{CVD}^{(3)}$. Oxidative stress and inflammatory reactions play important roles in the development of atherosclerosis ${ }^{(4,5)}$.

Carotenoids, which are abundant in many fruits and vegetables, are fat-soluble and antioxidant phytochemicals that efficiently quench singlet oxygen and free radicals ${ }^{(6)}$. In vitro studies suggest that carotenoids can inhibit the production of reactive oxygen species and the oxidative damaging activities of oxidised LDL involved in the development of atherosclerosis $^{(7,8)}$. A 7-year follow-up study in 840 middle-aged men in Finland suggested that high serum concentrations of lycopene, $\alpha$-carotene and $\beta$-carotene may exert a protective effect against early atherosclerosis ${ }^{(9)}$. In Asia, Ito et $a l^{(10)}$ found that high serum levels of carotenoids, such as $\alpha$ - and $\beta$-carotenes and lycopene, were associated with lower levels of cardiovascular mortality in a 2-year follow-up study of 1190 men and
1871 women in Japan. Similar results were found in other studies $^{(6,8,11,12)}$; however, the associations may have varied among different carotenoids in these studies. Zou et al. ${ }^{(6)}$ and Koh et $a l .{ }^{(12)}$ found that lutein and $\beta$-cryptoxanthin were protective and that $\alpha$-carotene, $\beta$-carotene and lycopene were not; these findings differ from those of other studies $(7,9,10,13)$. Because the serum concentrations of carotenoids and the predominant types of carotenoids among various populations vary greatly owing to their differing dietary patterns ${ }^{(8,10,12)}$, the associations found in other populations may not be generalisable to the Chinese population. Few studies have examined and compared the associations between the levels of various circulating carotenoids and early atherosclerosis or CVD in the Chinese population (all $n<1000$ subjects) $^{(6,12,14)}$. In addition, most of the studies did not adjust for dietary factors, such as the content of energy, fibre, fat and cholesterol, which might be associated with both serum carotenoid levels and the severity of atherosclerosis or $\mathrm{CVD}^{(15-18)}$. Moreover, although previously conducted clinical trials showed that

\footnotetext{
Abbreviations: CCA, common carotid artery; IMT, intima-media thickness.
}

* Corresponding author: Professor Y.-M. Chen, fax +86 20 87330446, email chenyum@mail.sysu.edu.cn

$\dagger$ These authors contributed equally to this work. 
supplementation with $\beta$-carotene may increase the risk of CVD death $^{(19)}$, the supplemental doses were much larger than the levels of dietary intake in the study population. Therefore, the effect of carotenoids under daily condition is inconclusive. Further study is needed to assess the associations between the levels of carotenoids and early atherosclerosis in Chinese people.

Given that the intima-media thickness (IMT) of the carotid artery is a sensitive and non-invasive surrogate for CVD and a mechanistic marker of subclinical atherosclerosis ${ }^{(20)}$, we performed this cross-sectional study to examine the hypothesis that higher serum levels of individual and total carotenoids would be beneficial to early atherosclerosis as measured by the carotid IMT in a middle-aged and elderly Chinese population.

\section{Methods}

In this cross-sectional study with a convenience sample for the Guangzhou Nutrition \& Health Study, 3169 subjects between 50 and 75 years of age were recruited by means of advertisements, health talks and referrals from communities in Guangzhou, China, between October 2008 and June 2010. The participants were required to have lived in Guangzhou for at least 5 years. We excluded participants who reported confirmed diagnoses of cancer, CHD, diabetes, renal failure, stroke, Alzheimer's disease or dementia. In addition, 801 participants were newly recruited using the same selection criteria and recruitment methods between February 2013 and August 2013. Finally, 3707 (1171 men and 2536 women) and 2947 (934 men and 2013 women) participants were included in the analyses of dietary and blood carotenoids (Fig. 1). The study protocol was

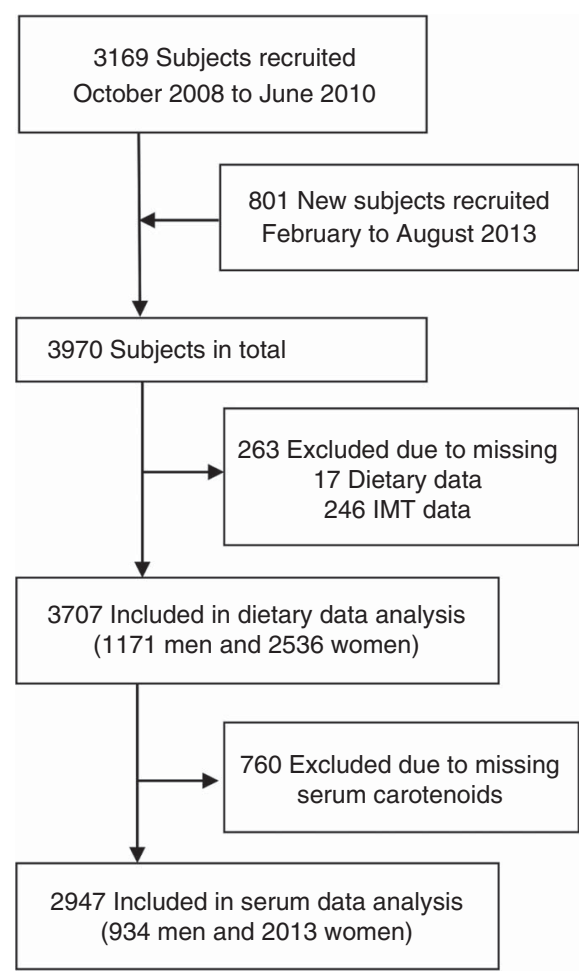

Fig. 1. Flow diagram of subject selection. IMT, intima-media thickness. approved by the Medical Ethics Committee of Sun Yat-sen University, and written informed consent was obtained from all participants.

\section{Data collection}

Measurements of serum carotenoids. Venous blood samples of the enrolled participants were collected after overnight fasting separately. Serum was separated after centrifugation at $4^{\circ} \mathrm{C}$ within $2 \mathrm{~h}$ and stored at $-80^{\circ} \mathrm{C}$ until analysis. Reversedphase HPLC was used to assay the levels of $\alpha$-carotene, $\beta$-carotene, $\beta$-cryptoxanthin, lycopene and lutein/zeaxanthin following the method of Burri et al. ${ }^{(21)}$ by the same researchers between 2012 and 2014. First, $200 \mu \mathrm{l}$ of each serum sample was mixed with $500 \mu$ l of ethanol-butylated hydroxytoluene solution containing $\alpha$-tocopherol acetate as the internal standard. The hexane layer was separated, and the residue was dissolved in $200 \mu \mathrm{l}$ of mobile phase B (acetonitrile-methanoltetrahydrofuran-ammonium acetate 55:35:5:5, v/v). We detected peaks at wavelengths of $450 \mathrm{~nm}$ for the carotenoids with a Waters 2998 diode-array detector (Waters). The combined concentration of lutein and zeaxanthin was used for these analyses. The day-to-day CV were approximately $7.8 \%$ for $\alpha$-carotene, $8.6 \%$ for $\beta$-carotene, $9.7 \%$ for $\beta$-cryptoxanthin, $10 \cdot 6 \%$ for lycopene and $8.0 \%$ for lutein + zeaxanthin.

Intima-media thickness assessment. The IMT was measured bilaterally at the far wall of the artery by using a high-resolution 7·0- to $12 \cdot 0-\mathrm{MHz}$ linear-array transducer system (Aplio; Toshiba) using a predetermined, standardised scanning protocol according to relevant guidelines ${ }^{(22)}$. We determined the IMT values of two 10-mm segments: the distal segment of the common carotid artery (CCA) and the bifurcation segment ${ }^{(23)}$. The wall thickness was measured manually with computer assistance using electronic callipers. B-mode images were recorded by professionals who were blinded to the identity of the subject and to all other study parameters. The site-specific reliability coefficients based on tests and retests in ninety-one randomly selected subjects on the same day were 0.72 and 0.68 for the mean carotid IMT at the far wall of the CCA and the carotid bifurcation segment, respectively. In our analysis, the IMT were calculated as the mean values from the right and left sides $^{(24)}$.

Assessment of covariates. The face-to-face interviews were conducted by trained interviewers using a structured questionnaire. Information was collected on the participants' socio-demographic characteristics, medications, lifestyle factors, history of disease, menopausal status (for women), dietary intake and physical activity. Physical activity was estimated using a nineteen-item physical activity questionnaire. The participants' usual dietary intake was assessed with a validated quantitative FFQ that included seventy-nine items ${ }^{(25)}$. Their daily intake of energy and nutrients was calculated on the basis of the Chinese Food Composition Table 2004 ${ }^{(26)}$.

The BMI was calculated as the participant's weight in $\mathrm{kg}$ divided by the square of his or her height in $\mathrm{m}$. Two consecutive blood pressure measurements were taken from the left arm, and the mean was used for subsequent analyses. 
Venous blood samples were collected after overnight fasting. The serum levels of total cholesterol, TAG, LDL-cholesterol, HDL-cholesterol and fasting blood glucose were measured. A detailed description of the measurement methods was published previously ${ }^{(24)}$.

\section{Statistical analysis}

The data from men and women were analysed both collectively and separately. Logarithmic transformation was used for the energy intake, and square root transformation was applied to the other dietary factors. The comparisons between men and women were performed with $t$ tests for continuous variables and $\chi^{2}$ tests for categorical variables. Total energy intake was adjusted for other dietary intakes of foods, nutrients and carotenoids before further analyses by using the residual method. The subjects were classified into quartiles according to their serum carotenoid concentrations or dietary consumptions of carotenoids. Univariate and multivariate analyses of variance were used to compare the mean IMT among the subjects in the serum or dietary carotenoid concentration quartiles. In the multivariate ANCOVA, adjustments were made for age, BMI, education level, family income, alcohol consumption, smoking status, level of physical activity and dietary intake of energy, and energy-adjusted intakes of total fat, saturated fatty acids, ratio of unsaturated fat to saturated fat, cholesterol and fibre. We further adjusted for blood pressure and plasma lipid level to determine the extent to which these intermediate variables could explain the association between carotenoid levels and the $\mathrm{IMT}^{(27)}$. The Bonferroni test was used to adjust for multiple comparisons in the pair-wise comparisons. Percentage mean difference was calculated as ' $(Q 4-Q 1) / Q 1 \times 100 \%$ '. We performed interaction analyses between the serum carotenoid concentration quartiles and the demographic and socioeconomic factors (age, sex, education level, household income, smoking and use of alcohol; $\alpha=0.05$ divided by the number of tests). The analyses were performed with SPSS 17.0 for Windows (SPSS, Inc.). Statistical significance was inferred for two-tailed $P$ values of $<0 \cdot 05$.

\section{Results}

\section{Characteristics of the study participants}

A total of 2947 participants (934 men and 2013 women) completed blood carotenoid measurements, dietary survey and carotid IMT tests were included in the serum carotenoid analysis. The characteristics of the participants are shown in Table 1. The mean age was 57.6 (SD 5.7) years for women and 60.6 (sD 6.6) years for the men. The IMT at the two studied sites was higher in men than in women $(P<0 \cdot 001)$. Women had higher values of dietary intake of fibre, fat and cholesterol; higher serum levels of HDL-cholesterol, LDL-cholesterol and serum carotenoids; and lower TAG and fasting glucose concentrations than the men.

The 3707 (1171 men and 2536 women) participants with dietary data and IMT data were included in the analysis of association between dietary carotenoid consumption and carotid IMT values. The mean values of carotenoids intakes of total participants were $1.31 \mathrm{mg} / \mathrm{d}$ for $\alpha$-carotene, $7.55 \mathrm{mg} / \mathrm{d}$ for $\beta$-carotene, $6.23 \mathrm{mg} / \mathrm{d}$ for lutein + zeaxanthin, $0.13 \mathrm{mg} / \mathrm{d}$ for $\beta$-cryptoxanthin $1.69 \mathrm{mg} / \mathrm{d}$ for lycopene and $16.87 \mathrm{mg} / \mathrm{d}$ for them combined in the total subjects (online Supplementary Table S1). The main food sources of dietary total carotenoids were dark green vegetables $(44.7 \%)$, carrots, peppers and tomatoes $(21.2 \%)$, vegetable melons, eggplant and radish $(20 \cdot 2 \%)$, and total fruit (7.89\%) (online Supplementary Table S2).

\section{Interaction analyses}

We explored the interaction between the serum carotenoid concentration quartiles and the demographic and socioeconomic factors (age, sex, education level, household income, smoking and use of alcohol) on the IMT levels. No significant interactions were found after adjusting for number of interaction analyses ( $P_{\text {interaction }}$ range: 0.033 to $0.960 ; \alpha=0.05$ / 36 tests) (online Supplementary Table S3). In the main text, we presented the results only in the combined sample of men and women.

\section{The association between serum carotenoids and carotid intima-media thickness}

Overall, we observed an inverse, dose-dependent association between the serum carotenoid concentrations and the mean IMT of each segment in both the univariate and multi-variable analyses of the whole sample (Table 2). In the univariate analysis, the means of carotid IMT in quartile 4 of serum carotenoid concentrations were $0.041-0.068 \mathrm{~mm}$ lower for CCA IMT and 0.038-0.086 mm lower for BIF IMT than those of quartile 1 (all $P_{\text {differences }}$ and $\left.P_{\text {trends }}<0 \cdot 001\right)$. After adjustment for demographic, socio-economic and lifestyle factors, the favourable associations of all of the studied carotenoids (except for lycopene) remained to be significant at the CCA and at the bifurcation segment $(P<0.001$ to 0.013$)$. The absolute mean differences in the IMT of the CCA between the participants in the extreme quartiles of serum carotenoid concentrations were $0.034 \mathrm{~mm} \quad(\alpha$-carotene $), \quad 0.037 \mathrm{~mm} \quad(\beta$-carotene $), 0.032 \mathrm{~mm}$ (lutein + zeaxanthin), $\quad 0.030 \mathrm{~mm}$ ( $\beta$-cryptoxanthin), $0.015 \mathrm{~mm}$ (lycopene) and $0.035 \mathrm{~mm}$ (total carotenoids); the corresponding values of the mean differences in the IMT at the bifurcation segment were $0.025,0.053,0.043,0.050,0.011$ and $0.042 \mathrm{~mm}$, respectively, for the above carotenoids. Similar results were revealed after further adjustment for blood pressure, glucose and lipid levels (online Supplementary Table S4). Among the studied carotenoids, $\beta$-carotene had the strongest association, followed by total carotenoids, lutein + zeaxanthin, $\alpha$-carotene and $\beta$-cryptoxanthin, whereas lycopene had the weakest association.

The association between dietary carotenoids and intima-media thickness

We also explored the associations between dietary intake of the carotenoids and the IMT values (Table 3). Significant beneficial 
Table 1. Characteristics of the study participants

(Mean values and standard deviations; numbers and percentages)

\begin{tabular}{|c|c|c|c|c|c|c|}
\hline \multirow[b]{2}{*}{ Characteristics } & \multicolumn{2}{|c|}{ Women } & \multicolumn{2}{|c|}{ Men } & \multicolumn{2}{|c|}{ Total } \\
\hline & Mean & SD & Mean & SD & Mean & SD \\
\hline$n$ & \multicolumn{2}{|c|}{2013} & \multicolumn{2}{|c|}{934} & \multicolumn{2}{|c|}{2947} \\
\hline Age (years) & $57 \cdot 6$ & $5 \cdot 7$ & $60.6 \ddagger$ & 6.6 & 58.6 & $6 \cdot 2$ \\
\hline $\mathrm{BMI}\left(\mathrm{kg} / \mathrm{m}^{2}\right)$ & $23 \cdot 1$ & $3 \cdot 1$ & $23 \cdot 7 \ddagger$ & 2.9 & 23.3 & $3 \cdot 1$ \\
\hline Waist circumference $(\mathrm{cm})$ & 81.9 & 8.9 & $86 \cdot 1 \ddagger$ & 8.6 & 83.3 & 9 \\
\hline Systolic blood pressure (mmHg) & $122 \cdot 9$ & $17 \cdot 6$ & $126 \cdot 7 \ddagger$ & $17 \cdot 8$ & $124 \cdot 1$ & $17 \cdot 7$ \\
\hline Diastolic blood pressure $(\mathrm{mmHg})$ & $76 \cdot 4$ & $10 \cdot 4$ & $79 \cdot 5 \ddagger$ & $10 \cdot 8$ & $77 \cdot 4$ & $10 \cdot 6$ \\
\hline \multicolumn{7}{|l|}{ Education } \\
\hline$\leq 6$ years & \multirow{2}{*}{\multicolumn{2}{|c|}{157}} & & & & \\
\hline$n$ & & & \multicolumn{2}{|c|}{$47 \ddagger$} & \multicolumn{2}{|c|}{204} \\
\hline$\%$ & \multicolumn{2}{|c|}{$7 \cdot 8$} & \multicolumn{2}{|c|}{$5 \cdot 0$} & \multicolumn{2}{|c|}{6.9} \\
\hline \multicolumn{7}{|l|}{$6-12$ years } \\
\hline$n$ & \multicolumn{2}{|c|}{1419} & & & & \\
\hline$\%$ & & & & & & \\
\hline$>12$ years & & & & & & \\
\hline$n$ & & & & & & \\
\hline$\%$ & & & & & & \\
\hline Family monthly income (Yuan/per & & & & & & \\
\hline$<1500$ & & & & & & \\
\hline$n$ & & & & & & \\
\hline$\%$ & & & & & & \\
\hline $1500-3000$ & & & & & & \\
\hline$n$ & & & & & & \\
\hline$\%$ & & & & & & \\
\hline$>3000$ & & & & & & \\
\hline$n$ & & & & & & \\
\hline$\%$ & & & & & & \\
\hline Alcohol drinker & & & & & & \\
\hline$n$ & & & & & & \\
\hline$\%$ & & & & & & \\
\hline Smoker & & & & & & \\
\hline$n$ & & & & & & \\
\hline$\%$ & & & & & & \\
\hline Physical activities (MET.h/d) & $24 \cdot 3$ & $6 \cdot 1$ & $23 \cdot 7$ & $7.0 \dagger$ & $24 \cdot 1$ & $6 \cdot 4$ \\
\hline Dietary intakes & & & & & & \\
\hline Energy $(\mathrm{kJ} / \mathrm{d})$ & 7393 & 2088 & 8401 & 2385 & 7715 & 2234 \\
\hline Energy (kcal/d) & 1767 & 499 & $2008 \ddagger$ & 570 & 1844 & 534 \\
\hline Fat $(\mathrm{g} / \mathrm{d})$ & $46 \cdot 6$ & $10 \cdot 5$ & $43.9 \ddagger$ & $10 \cdot 1$ & $45 \cdot 7$ & $10 \cdot 5$ \\
\hline Fibre $(g / d)$ & 11.5 & 3.5 & $10.2 \ddagger$ & 3.0 & $11 \cdot 1$ & $3 \cdot 4$ \\
\hline Cholesterol (mg/d) & 393 & 178 & $371 \ddagger$ & 161 & 386 & 173 \\
\hline SFA $(g / d)$ & 12.9 & 3.5 & $12 \cdot 1 \ddagger$ & $3 \cdot 3$ & $12 \cdot 6$ & 3.5 \\
\hline (MUFA + PUFA)/SFA & $2 \cdot 24$ & 0.38 & $2 \cdot 27$ & 0.34 & 2.25 & 0.37 \\
\hline Total vegetable and fruit $(\mathrm{g} / \mathrm{d})$ & 532 & 279 & $495 \ddagger$ & 270 & 520 & 277 \\
\hline Biochemical indicators $(\mathrm{mmol} / \mathrm{l})$ & & & & & & \\
\hline Total cholesterol & $5 \cdot 65$ & 1.11 & $5 \cdot 24 \ddagger$ & 1.04 & 5.52 & 1.1 \\
\hline LDL-cholesterol & 3.65 & 0.92 & $3.42 \ddagger$ & 0.86 & 3.58 & 0.91 \\
\hline HDL-cholesterol & 1.46 & 0.34 & $1.27 \ddagger$ & 0.31 & 1.40 & 0.34 \\
\hline TAG & 1.54 & 1.22 & $1.66 \dagger$ & 1.40 & 1.58 & 1.28 \\
\hline Fasting glucose & 4.80 & 1.03 & $5.05 \ddagger$ & 1.17 & 4.88 & 1.09 \\
\hline Carotenoids $(\mu \mathrm{mol} / \mathrm{l})$ & & & & & & \\
\hline$a$-Carotene & 0.078 & 0.060 & $0.064 \ddagger$ & 0.048 & 0.073 & 0.057 \\
\hline$\beta$-Carotene & 0.590 & 0.391 & $0.404 \ddagger$ & 0.301 & 0.531 & 0.375 \\
\hline Lutein + zeaxanthin & 0.707 & 0.351 & $0.637 \ddagger$ & 0.318 & 0.684 & 0.343 \\
\hline$\beta$-Cryptoxanthin & 0.178 & 0.153 & $0.123 \ddagger$ & 0.102 & 0.161 & 0.141 \\
\hline Lycopene & 0.200 & 0.132 & $0.161 \ddagger$ & 0.117 & 0.188 & 0.129 \\
\hline Total carotenoids & $1 \cdot 754$ & 0.818 & $1.390 \ddagger$ & 0.667 & 1.638 & 0.791 \\
\hline Carotid IMT (mm) & & & & & & \\
\hline CCA & 0.685 & 0.130 & $0.746 \ddagger$ & 0.172 & 0.704 & 0.148 \\
\hline BIF & 0.889 & 0.199 & $0.980 \ddagger$ & 0.226 & 0.918 & 0.212 \\
\hline
\end{tabular}

MET, metabolic equivalent; IMT, intima-media thickness; CCA, common carotid artery segment; BIF, bifurcation segment.

Difference between sex groups: $\uparrow P<0.05 ; \ddagger P<0.01$.

associations were observed at the CCA IMT in both the univariate and multivariate models (except for lycopene), although the associations of dietary carotenoids tended to be attenuated as compared with those of serum carotenoids. However, no significant associations were found at the BIF IMT for all studied dietary carotenoids (all $P_{\text {difference }}$ and $P_{\text {trend }}>0.05$ ). 
Table 2. Comparison of the mean intima-media thickness ( $\mathrm{mm}$ ) at the common carotid artery segment (CCA) and bifurcation segment (BIF) among quartiles (Q) of serum carotenoid concentrations ( $n$ 2947, 934 men and 2013 women)

(Mean values and standard deviations; mean values with their standard errors)

\begin{tabular}{|c|c|c|c|c|c|c|c|c|c|c|c|c|}
\hline \multirow[b]{3}{*}{ Variables } & \multicolumn{8}{|c|}{ Quartiles by each carotenoid } & \multirow[b]{3}{*}{ Diff. (mm) } & \multirow[b]{3}{*}{ \%Diff. (\%) } & \multirow[b]{3}{*}{$P_{\text {difference }}$} & \multirow[b]{3}{*}{$P_{\text {trend }}$} \\
\hline & \multicolumn{2}{|c|}{ Q1 } & \multicolumn{2}{|c|}{ Q2 } & \multicolumn{2}{|c|}{ Q3 } & \multicolumn{2}{|c|}{ Q4 (highest) } & & & & \\
\hline & Mean & $\mathrm{SD}$ & Mean & $\mathrm{SD}$ & Mean & $\mathrm{SD}$ & Mean & SD & & & & \\
\hline \multicolumn{13}{|l|}{ a-Carotene } \\
\hline Median $(\mu \mathrm{mol} / \mathrm{l})$ & & & & 028 & & & 0.07 & & & & & \\
\hline $\mathrm{CCA}, \mathrm{M} 1^{*}$ & 0.729 & 0.179 & $0.708 \dagger$ & 0.137 & $0.696 \ddagger$ & 0.142 & $0.684 \ddagger \S \S$ & 0.123 & -0.045 & $-6 \cdot 17$ & $<0.001$ & $<0.001$ \\
\hline CCA, M2\| & & & & & & & & & -0.034 & $-4 \cdot 70$ & $<0.001$ & $<0.001$ \\
\hline Mean & & & & 707 & & $9 \ddagger$ & 0.69 & & & & & \\
\hline SEM & & & & 005 & & & 0.0 & & & & & \\
\hline $\mathrm{BIF}, \mathrm{M} 1^{*}$ & 0.938 & 0.206 & 0.923 & 0.231 & 0.912 & 0.213 & $0.900 \ddagger$ & 0.195 & -0.038 & -4.05 & 0.005 & $<0.001$ \\
\hline BIF, M2\|l & & & & & & & & & -0.025 & $-2 \cdot 69$ & 0.118 & 0.013 \\
\hline Mean & & & & 918 & & & 0.90 & & & & & \\
\hline SEM & & & & 007 & & & 0.00 & & & & & \\
\hline$\beta$-Carotene & & & & & & & & & & & & \\
\hline Median $(\mu \mathrm{mol} / \mathrm{l})$ & & & & 384 & & & $1 \cdot 12$ & & & & & \\
\hline $\mathrm{CCA}, \mathrm{M} 1^{*}$ & 0.738 & 0.149 & $0.709 \ddagger$ & 0.172 & $0.701 \ddagger$ & 0.134 & $0.670 \ddagger \S \S$ & 0.123 & -0.068 & -9.21 & $<0.001$ & $<0.001$ \\
\hline CCA, M2\| & & & & & & & & & -0.037 & $-5 \cdot 12$ & $<0.001$ & $<0.001$ \\
\hline Mean & & & & 708 & & & 0.685 & & & & & \\
\hline SEM & & & & 05 & & & 0.00 & & & & & \\
\hline $\mathrm{BIF}, \mathrm{M} 1^{*}$ & $0.962 \ddagger$ & 0.221 & $0.922 \ddagger$ & 0.212 & $0.912 \ddagger$ & 0.204 & $0.876 \mp \S \S$ & 0.202 & -0.086 & -8.94 & $<0.001$ & $<0.001$ \\
\hline BIF, M2\| & & & & & & & & & -0.053 & -5.63 & $<0.001$ & $<0.001$ \\
\hline Mean & & & & 920 & & & 0.888 & & & & & \\
\hline SEM & & & & 007 & & & 0.00 & & & & & \\
\hline Lutein + zeaxanthin & & & & & & & & & & & & \\
\hline Median $(\mu \mathrm{mol} / \mathrm{l})$ & & & & 366 & & & 0.75 & & & & & \\
\hline $\mathrm{CCA}, \mathrm{M} 1^{\star}$ & 0.725 & 0.143 & 0.714 & 0.175 & $0.702 \dagger$ & 0.138 & $0.676 \neq \S \S$ & 0.126 & -0.049 & $-6 \cdot 76$ & $<0.001$ & $<0.001$ \\
\hline CCA, M2\| & & & & & & & & & -0.032 & -4.46 & $<0.001$ & $<0.001$ \\
\hline Mean & & & & 712 & & & 0.685 & & & & & \\
\hline SEM & & & & 005 & & & 0.00 & & & & & \\
\hline $\mathrm{BIF}, \mathrm{M} 1^{*}$ & 0.948 & $0 \cdot 212$ & 0.924 & 0.213 & $0.907 \ddagger$ & 0.193 & $0.892 \ddagger \S$ & 0.225 & -0.056 & $-5 \cdot 91$ & $<0.001$ & $<0.001$ \\
\hline BIF, M2\|l & & & & & & & & & -0.043 & -4.57 & $<0.001$ & $<0.001$ \\
\hline Mean & & & & 922 & & & 0.89 & & & & & \\
\hline SEM & & & & 007 & & & 0.0 & & & & & \\
\hline$\beta$-Cryptoxanthin & & & & & & & & & & & & \\
\hline Median $(\mu \mathrm{mol} / \mathrm{l})$ & & & & 055 & & & 0.18 & & & & & \\
\hline $\mathrm{CCA}, \mathrm{M} 1^{*}$ & 0.731 & 0.150 & 0.721 & $0.179 \neq \S \S$ & 0.682 & $0.130 \pm \S \S$ & 0.684 & 0.118 & -0.047 & -6.43 & $<0.001$ & $<0.001$ \\
\hline CCA, M2\| & & & & & & & & & -0.030 & $-4 \cdot 16$ & $<0.001$ & $<0.001$ \\
\hline Mean & & & & 719 & & $36 \ddagger$ & 0.692 & & & & & \\
\hline SEM & & & & 005 & & & 0.0 & & & & & \\
\hline $\mathrm{BIF}, \mathrm{M} 1^{*}$ & 0.958 & 0.223 & $0.928 \dagger$ & 0.204 & $0.899 \ddagger \S$ & 0.224 & $0.887 \ddagger \S \S$ & 0.188 & -0.071 & $-7 \cdot 41$ & $<0.001$ & $<0.001$ \\
\hline BIF, M2\| & & & & & & & & & -0.050 & $-5 \cdot 29$ & $<0.001$ & $<0.001$ \\
\hline Mean & & & & 926 & & & 0.89 & & & & & \\
\hline SEM & & & & 007 & & & 0.0 & & & & & \\
\hline Lycopene & & & & & & & & & & & & \\
\hline Median $(\mu \mathrm{mol} / \mathrm{l})$ & & & & 075 & & & 0.15 & & & & & \\
\hline $\mathrm{CCA}, \mathrm{M} 1^{*}$ & 0.731 & 0.176 & $0.701 \dagger$ & 0.140 & $0.696 \ddagger$ & 0.130 & $0.690 \ddagger$ & 0.137 & -0.041 & $-5 \cdot 61$ & $<0.001$ & $<0.001$ \\
\hline CCA, M2\| & & & & & & & & & -0.015 & -2.09 & 0.087 & 0.061 \\
\hline Mean & & & & 700 & & & 0.70 & & & & & \\
\hline SEM & & & & 005 & & & 0.0 & & & & & \\
\hline $\mathrm{BIF}, \mathrm{M} 1^{*}$ & 0.942 & 0.235 & 0.920 & 0.207 & $0.911 \ddagger$ & 0.220 & $0.899 \ddagger$ & 0.180 & -0.043 & -4.56 & 0.001 & $<0.001$ \\
\hline BIF, M2\|l & & & & & & & & & -0.011 & $-1 \cdot 19$ & 0.777 & 0.311 \\
\hline Mean & & & & 920 & & & 0.9 & & & & & \\
\hline SEM & & & & 007 & & & 0.0 & & & & & \\
\hline Total carotenoid & & & & & & & & & & & & \\
\hline Median $(\mu \mathrm{mol} / \mathrm{l})$ & & & & 671 & & & $1 \cdot 3$ & & & & & \\
\hline $\mathrm{CCA}, \mathrm{M} 1^{*}$ & 0.733 & 0.149 & 0.718 & 0.177 & $0.693 \ddagger \S$ & 0.126 & $0.673 \ddagger \S \S$ & 0.125 & -0.060 & $-8 \cdot 19$ & $<0.001$ & $<0.001$ \\
\hline CCA, M2\| & & & & & & & & & -0.035 & -4.85 & $<0.001$ & $<0.001$ \\
\hline Mean & & & & 712 & & $99+$ & 0.686 & & & & & \\
\hline SEM & & & & 005 & & & 0.00 & & & & & \\
\hline $\mathrm{BIF}, \mathrm{M} 1^{*}$ & 0.956 & 0.221 & 0.931 & 0.217 & $0.901 \ddagger \S$ & 0.204 & $0.884 \ddagger \S \S$ & 0.198 & -0.072 & -7.53 & $<0.001$ & $<0.001$ \\
\hline BIF, M2\| & & & & & & & & & -0.042 & -4.46 & $<0.001$ & $<0.001$ \\
\hline Mean & & & & 925 & & $4 \ddagger$ & 0.89 & & & & & \\
\hline SEM & & & & 007 & & & 0.0 & & & & & \\
\hline
\end{tabular}

M1, model 1; M2, model 2; Diff., absolute difference $=\mathrm{Q} 4-\mathrm{Q} 1 ; \%$ Diff., percentage difference $=(\mathrm{Q} 4-\mathrm{Q} 1) / \mathrm{Q} 1 \times 100 \% ; P_{\text {difference, }} P$ values for difference among quartiles. Compared with Q1: $\dagger P<0.05, \ddagger P<0.01$. Compared with Q2, $§ P<0.05, \S \S P<0.01$. The Bonferroni method was used to adjust for multiple comparisons.

* M1: ANOVA.

॥ M2: ANCOVA, adjusted for age, sex, BMI, education status, family income, alcohol drinking status, smoking status, physical activities, dietary intakes of energy and energyadjusted total fat, SFA, ratio of unsaturated fat:SFA, cholesterol and fibre. 
Table 3. Comparison of the mean intima-media thickness $(\mathrm{mm})$ at the common carotid artery segment (CCA) and bifurcation segment (BIF) among quartiles $(\mathrm{Q})$ of dietary carotenoids ( $n 3707,1171$ men and 2536 women)

(Mean values and standard deviations; mean values with their standard errors)

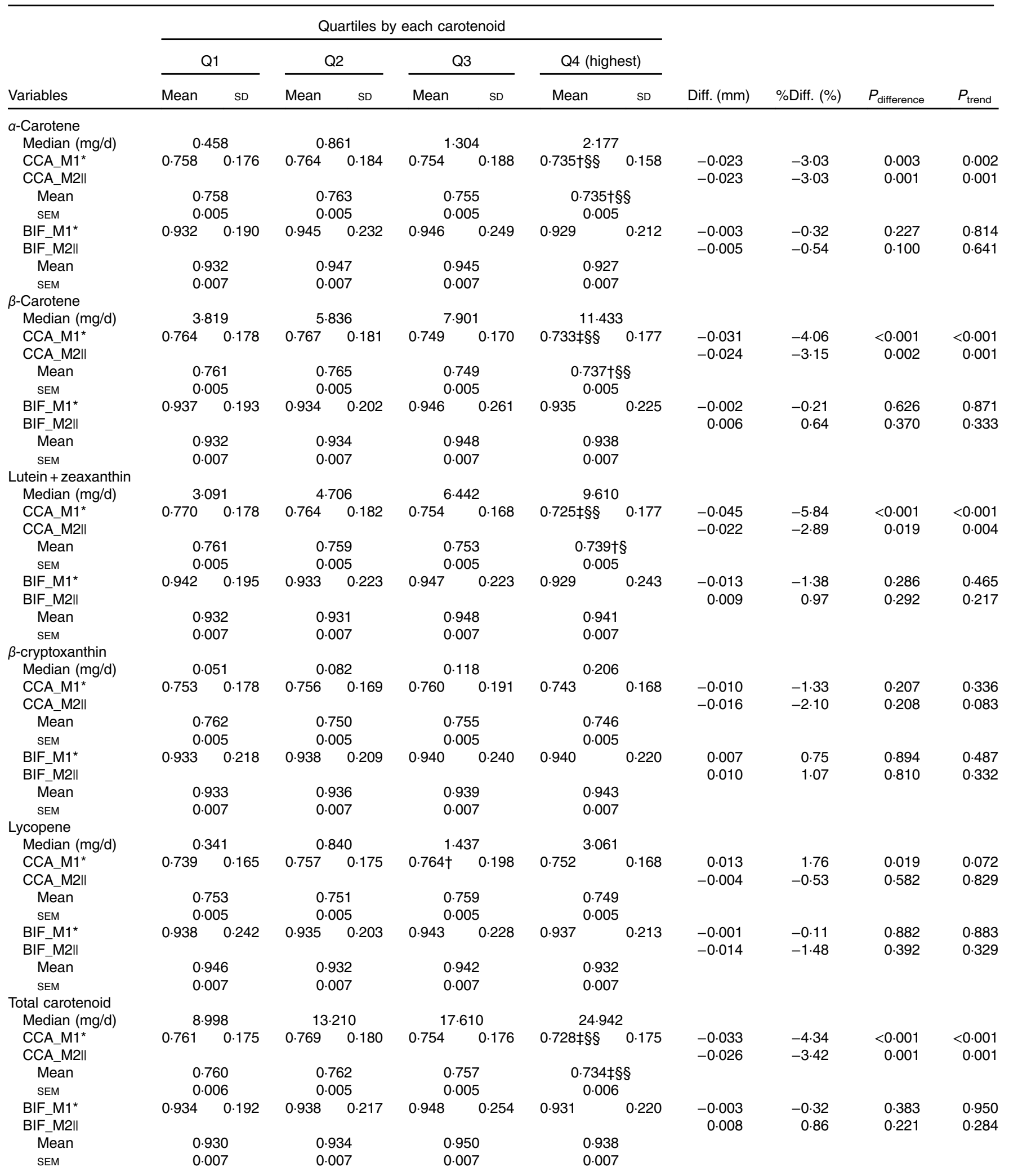

M1, model; M2, model 2; Diff., absolute difference $=\mathrm{Q} 4-\mathrm{Q} 1 ; \%$ Diff., percentage difference $=(\mathrm{Q} 4-\mathrm{Q} 1) / \mathrm{Q} 1 \times 100 \% ; P_{\text {difference, }} P$ values for difference among quartiles.

Compared with Q1: † $P<0.05, \ddagger P<0.01$. Compared with Q2, $§ P<0.05, \S \S P<0.01$. The Bonferroni method was used to adjusted for multiple comparisons.

* M1: ANOVA.

॥ M2: ANCOVA, adjusted for age, sex, BMI, education status, family income, alcohol drinking status, smoking status, physical activities, dietary intakes of energy and energyadjusted total fat, SFA, ratio of unsaturated fat:SFA, cholesterol and fibre. 


\section{Discussion}

In this large community-based study, the serum concentrations and dietary consumptions of total carotenoids and of $\alpha$-carotene, $\beta$-carotene, lutein + zeaxanthin and $\beta$-cryptoxanthin, but not lycopene, were graded and were found to be inversely associated with the carotid IMT in middle-aged and elderly Chinese women and men.

Many studies have examined the association between carotenoids in blood or diet and carotid IMT or atherosclerosis ${ }^{(28)}$. In a randomised clinical trial in 144 subjects with subclinical atherosclerosis, one-year supplementations with daily doses of $20 \mathrm{mg}$ of lutein or $20 \mathrm{mg}$ of lutein plus $20 \mathrm{mg}$ of lycopene ( $v$. placebo) significantly decreased mean carotid IMT by $0.035 \mathrm{~mm}$ $(P=0.042)$ and $0.073 \mathrm{~mm}(P<0.001)^{(29)}$. Three prospective studies showed significant and inverse associations of blood carotenoid(s) with 7-year IMT changes in 840 middle-aged Finnish men ${ }^{(9)}$, with 18-month IMT progression in 573 middleaged American men and women ${ }^{(8,11)}$, with the 5 -year incidence of carotid atherosclerotic lesions in 392 Italian men and women aged 45-65 years ${ }^{(30)}$. The favourable associations between blood carotenoids and early atherosclerosis were observed in two case-control studies comprising 125 cases and 107 controls aged $45-68$ years $^{(6)}$ and in forty pairs of cases and controls ${ }^{(14)}$ in Beijing, China. Similar results were also noted in several crosssectional studies of blood carotenoid analyses, including 392 Italian men and women ${ }^{(30)}, 1212$ elderly men (aged 61-80 years) ${ }^{(31)}$, 1028 middle-aged men (aged 46-64 years) ${ }^{(32)}$ and 520 middle-aged men and women (aged 45-69 years) ${ }^{(33)}$ in eastern Finland. One cross-sectional study of the Atherosclerosis Risk in Communities Study showed that subjects with higher carotenoid consumption had a lower prevalence of plaques among 12,773 American participants aged 45-64 years $^{(34)}$. As indicated in a recent systemic review ${ }^{(28)}$, no significant favourable associations of blood carotenoids with IMT or atherosclerosis were observed in one cross-sectional study with 1187 French men and women aged 59-71 years ${ }^{(35)}$ and two case-control studies in 231 pairs of American adults ${ }^{(36)}$ and in 108 subjects with aortic atherosclerosis and controls in the Netherlands ${ }^{(7)}$. In this cross-sectional study with relatively large study size, we found a favourable association between the levels of carotenoids in both diet and serum and carotid IMT (particular at the CCA segment). The beneficial associations were largely consistent across different sex, age, education level, income status, smoking and alcohol drinking status (all $P_{\text {interaction }}>0.05 /$ number of tests), suggesting a good internal consistency. In summary, the findings from the majority of previous studies and our study supported the hypothesis that higher levels of dietary or blood carotenoids may be beneficial to IMT or early atherosclerosis in adults.

Generally, the strength of the studied associations of dietary carotenoids tended to be lower than those of serum carotenoids, possibly owing to greater random error in the long-term dietary recalls and large between-individual variations in bioavailability of dietary carotenoids ${ }^{(37-39)}$. Participants with the highest (v. lowest) quartile intake of total carotenoids $(24.9 v .9 .0 \mathrm{mg} / \mathrm{d})$ and vegetables ( $491 v .175 \mathrm{~g} / \mathrm{d}$ ) had significantly lower CCA IMT $(-3.42 \%$ (Table 3$)$ and $-3.29 \%$ (data not shown)). However, no significant association was observed between fruit intake and the IMT values in our study (data not shown). These results could be explained by the food sources of carotenoids, in which more than $90 \%$ of total carotenoids were provided by vegetables in this population (online Supplementary Table S2). Given that there are no endogenetic carotenoids in blood, the association of dietary data should have been underestimated in this study. As relatively low correlation coefficients were observed between the FFQ-based dietary data and in serum values of carotenoids, further interventional studies on the relationship between dietary intake and serum levels of carotenoids are needed to translate the serum values to the corresponding dietary intake of each individual carotenoid and carotenoid-rich food.

In this study, the association between the serum level of lycopene and the IMT was generally weaker than those between other kinds of carotenoids and the IMT. The reason for the difference between our results and those of other studies $^{(8,10,40)}$ may be the lower mean concentrations. Tomatoes are rich in lycopene, and Chinese people consume fewer tomatoes than people in many other countries. In our study, the mean serum concentration of lycopene was $0 \cdot 188 \mu \mathrm{mol} / 1$ in men and women combined, whereas the corresponding values were $0.371 \mu \mathrm{mol} / 1$ in $\operatorname{Japan}^{(10)}$ and $0.760 \mu \mathrm{mol} / 1$ in the $\mathrm{USA}^{(8)}$. Therefore, further study is needed to validate the association between lycopene and atherosclerosis in the Chinese population.

The possible mechanism of the protective activity of carotenoids has been extensively summarised in several recent reviews $^{(1,4,5,41,42)}$. By acting as antioxidants, carotenoids can quench singlet oxygen $\left({ }^{1} \mathrm{O}_{2}\right)$ and potentially reduce the toxic effects of reactive oxygen species, which indiscriminately destroy cell components ${ }^{(1)}$. On the other hand, oxidative modification of LDL in the subendothelial space plays a central role in atherogenesis by increasing the production of cytotoxic oxidatively modified LDL, which promotes endothelial dysfunction and the expression of adhesion molecules ${ }^{(4)}$. Many studies have shown that low concentrations of serum carotenoids appear to be associated with higher risks of metabolic syndrome status and its components ${ }^{(27,43,44)}$. We further adjusted for plasma lipid levels and blood pressure to assess to what extent these variables could explain the association between the carotenoid levels and the IMT values in our study. The associations between serum carotenoids and the IMT were slightly attenuated in women after further adjustment for these potential 'intermediate' variables. Therefore, our findings imply that the favourable effects of carotenoids on the IMT might be partially mediated by an improvement in blood pressure and in the plasma lipid levels. Carotenoids can also exhibit anti-inflammatory effects via the inhibition of C-reactive protein, leucocytes, fibrinogen and inflammatory cytokines ${ }^{(45)}$.

This study has several strengths. First, to the authors' knowledge, this is largest study that analysed the association between serum carotenoid levels and IMT. The large study size permitted us to obtain more precise results. Moreover, the objective biomarkers would ensure more accurate assessment of the internal exposure to carotenoids and better reflect the 
real effective dose in vivo ${ }^{(37-39)}$. Second, we assessed the associations of carotenoids and IMT both in dietary data and serum biomarkers, and obtained consistent results. In addition, similar results were observed among different carotenoids and across different groups of age, sex, education level and status of income, smoking and alcohol drinking. The good internal consistency would decrease the probability of a chance association. Third, we controlled for the covariates to avoid potential confounding.

However, this study also has several limitations. First, the cross-sectional design may have limitations in the identification of a causal relationship owing to the unclear time relationship between the carotenoid levels and the IMT measurements. To avoid the potential inverse causal relationship, we excluded all subjects with previously confirmed chronic diseases, such as cancer, CHD and stroke, which might have changed their dietary habits. Second, the study participants were recruited from urban areas instead of random sampling, which might potentially have led to a selection bias and limited the generalisability of our findings. However, the associations were not significantly modified by several demographic and socioeconomic factors, smoking and alcohol consumption, which suggested that the generalisability of our findings was not significantly affected by these factors. Third, 1023 subjects were excluded from the serum data analyses because of missing data; however, there were no significant differences in the characteristics between included and excluded participants, except income status (online Supplementary Table S5). Finally, we were unable to exclude the effects of many known or unknown co-existing nutritional factors on the levels of carotenoids in the blood.

In conclusion, we found that higher dietary and serum carotenoid levels were associated with lower carotid IMT values. Our findings suggest that elevated levels of dietary and circulating carotenoids may be of benefit to the prevention of early atherosclerosis. Further well-designed prospective studies are needed to confirm these findings in our population.

\section{Acknowledgements}

The authors thank the voluntary blood donors that participated in this study, and the authors are grateful for the help of other staff, postgraduates and undergraduates involved in data collection in this study.

This study was jointly supported by the National Natural Science Foundation of China (nos 81472965, 81372976, 81730090) and the 5010 Program for Clinical Researches (no. 2007032) by the Sun Yat-sen University, Guangzhou, China. The funders had no role in study design, data collection and analysis, decision to publish or preparation of the manuscript

Y.-M. C. conceived and designed the research; C. W. performed the statistical analysis; C. W. and Y. M.-C. wrote the paper; C. W., R. Q., Y. C. and W.-F. O. conducted the research; W.-H. L. and H.-B. L. revised the paper; and Y.-M. C. had primary responsibility for final content. All authors read and approved the final manuscript.

The authors declare that there are no conflicts of interest.

\section{Supplementary material}

For supplementary material/s referred to in this article, please visit https://doi.org/10.1017/S0007114517003932

\section{References}

1. Young AJ \& Lowe GM (2001) Antioxidant and prooxidant properties of carotenoids. Arch Biochem Biophys $\mathbf{3 8 5}$, $20-27$.

2. Mathers CD (2006) Projections of global mortality and burden of disease from 2002 to 2030. PLoS Med 3, 2011-2030.

3. Aatola H, Koivistoinen T, Hutri-Kahonen N, et al. (2010) Lifetime fruit and vegetable consumption and arterial pulse wave velocity in adulthood: the Cardiovascular Risk in Young Finns Study. Circulation 122, 2521-2528.

4. Tavakoli S \& Asmis R (2012) Reactive oxygen species and thiol redox signaling in the macrophage biology of atherosclerosis. Antioxid Redox Signal 17, 1785-1795.

5. Peter Libby MD (2012) History of discovery: inflammation in atherosclerosis. Arterioscler Thromb Vasc Biol 32, 2045-2051.

6. Zou Z, Xu X, Huang Y, et al. (2011) High serum level of lutein may be protective against early atherosclerosis: the Beijing atherosclerosis study. Atherosclerosis 219, 789-793.

7. Klipstein-Grobusch K, Launer LJ, Geleijnse JM, et al. (2000) Serum carotenoids and atherosclerosis. The Rotterdam Study. Atherosclerosis 148, 49-56.

8. Dwyer JH, Navab M, Dwyer KM, et al. (2001) Oxygenated carotenoid lutein and progression of early atherosclerosis : The Los Angeles Atherosclerosis Study. Circulation 103, 2922-2927.

9. Karppi J, Kurl S, Ronkainen K, et al. (2013) Serum carotenoids reduce progression of early atherosclerosis in the carotid artery wall among Eastern Finnish men. PLOS ONE 8, e64107.

10. Ito Y, Kurata M, Suzuki K, et al. (2006) Cardiovascular disease mortality and serum carotenoid levels: a Japanese populationbased follow-up study. J Epidemiol 16, 154-160.

11. Dwyer JH, Paul-Labrador MJ, Fan J, et al. (2004) Progression of carotid intima-media thickness and plasma antioxidants: the Los Angeles Atherosclerosis Study. Arterioscler Thromb Vasc Biol 24, 313-319.

12. Koh WP, Yuan JM, Wang R, et al. (2011) Plasma carotenoids and risk of acute myocardial infarction in the Singapore Chinese Health Study. Nutr Metab Carbiovasc Dis 21, 685-690.

13. Sesso HD, Buring JE, Norkus EP, et al. (2004) Plasma lycopene, other carotenoids, and retinol and the risk of cardiovascular disease in women. Am J Clin Nutr 79, 47-53.

14. Xu XR, Zou ZY, Huang YM, et al. (2012) Serum carotenoids in relation to risk factors for development of atherosclerosis. Clin Biochem 45, 1357-1361.

15. Wu H, Flint AJ, Qi Q, et al. (2015) Association between dietary whole grain intake and risk of mortality: two large prospective studies in US men and women. JAMA Intern Med 175, 373-384.

16. Muraki I, Wu H, Imamura F, et al. (2015) Rice consumption and risk of cardiovascular disease: results from a pooled analysis of 3 U.S. cohorts. Am J Clin Nutr 101, 164-172.

17. Michas G, Micha R \& Zampelas A (2014) Dietary fats and cardiovascular disease: putting together the pieces of a complicated puzzle. Atherosclerosis 234, 320-328.

18. Iso H, Kobayashi M, Ishihara J, et al. (2006) Intake of fish and $n 3$ fatty acids and risk of coronary heart disease among Japanese - The Japan Public Health Center-based (JPHC) Study Cohort I. Circulation 113, 195-202.

19. Vivekananthan DP, Penn MS, Sapp SK, et al. (2003) Use of antioxidant vitamins for the prevention of cardiovascular disease: meta-analysis of randomised trials. Lancet 361, 2017-2023. 
20. Lorenz MW, Markus HS, Bots ML, et al. (2007) Prediction of clinical cardiovascular events with carotid intima-media thickness: a systematic review and meta-analysis. Circulation 115, 459-467.

21. Burri BJ, Dopler-Nelson M \& Neidllinger TR (2003) Measurements of the major isoforms of vitamins $\mathrm{A}$ and $\mathrm{E}$ and carotenoids in the blood of people with spinal-cord injuries. J Chromatogr A 987, 359-366.

22. Roman MJ, Naqvi TZ, Gardin JM, et al. (2006) American Society of Echocardiography report. Clinical application of noninvasive vascular ultrasound in cardiovascular risk stratification: a report from the American Society of Echocardiography and the Society for Vascular Medicine and Biology. Vasc Med 11, 201-211.

23. Wang P, Chen YM, He LP, et al. (2012) Association of natural intake of dietary plant sterols with carotid intima-media thickness and blood lipids in Chinese adults: a crosssection study. PLOS ONE 7, e32736.

24. Zhang B, Chen YM, Huang LL, et al. (2008) Greater habitual soyfood consumption is associated with decreased carotid intima-media thickness and better plasma lipids in Chinese middle-aged adults. Atherosclerosis 198, 403-411.

25. Zhang CX \& Ho SC (2009) Validity and reproducibility of a food frequency questionnaire among Chinese women in Guangdong province. Asia Pac J Clin Nutr 18, 240-250.

26. Yang YX (2004) China Food Composition Table 2004. Beijing: Peking University Medical Press.

27. Ried K \& Fakler P (2011) Protective effect of lycopene on serum cholesterol and blood pressure: Meta-analyses of intervention trials. Maturitas 68, 299-310.

28. Hosseini B, Saedisomeolia A \& Skilton MR (2017) Association between micronutrients intake/status and carotid intima media thickness: a systematic review. J Acad Nutr Diet 117, 69-82.

29. Zou ZY, Xu XR, Lin XM, et al. (2014) Effects of lutein and lycopene on carotid intima-media thickness in Chinese subjects with subclinical atherosclerosis: a randomised, doubleblind, placebo-controlled trial. Br J Nutr 111, 474-480.

30. D'Odorico A, Martines D, Kiechl S, et al. (2000) High plasma levels of alpha- and beta-carotene are associated with a lower risk of atherosclerosis: results from the Bruneck study. Atherosclerosis 153, 231-239.

31. Karppi J, Kurl S, Laukkanen JA, et al. (2011) Plasma carotenoids are related to intima-media thickness of the carotid artery wall in men from eastern Finland. J Intern Med 270, 478-485.

32. Rissanen TH, Voutilainen S, Nyyssonen K, et al. (2003) Serum lycopene concentrations and carotid atherosclerosis: the Kuopio Ischaemic Heart Disease Risk Factor Study. Am J Clin Nutr 77, 133-138.
33. Rissanen T, Voutilainen S, Nyyssonen K, et al. (2000) Low plasma lycopene concentration is associated with increased intima-media thickness of the carotid artery wall. Arterioscler Thromb Vasc Biol 20, 2677-2681.

34. Kritchevsky SB, Tell GS, Shimakawa T, et al. (1998) Provitamin A carotenoid intake and carotid artery plaques: the Atherosclerosis Risk in Communities Study. Am J Clin Nutr $\mathbf{6 8}$, 726-733.

35. Bonithon-Kopp C, Coudray C, Berr C, et al. (1997) Combined effects of lipid peroxidation and antioxidant status on carotid atherosclerosis in a population aged 59-71 y: The EVA Study. Etude sur le Vieillisement Arteriel. Am J Clin Nutr 65, 121-127.

36. Iribarren C, Folsom AR, Jacobs DR Jr, et al. (1997) Association of serum vitamin levels, LDL susceptibility to oxidation, and autoantibodies against MDA-LDL with carotid atherosclerosis. A case-control study. The ARIC Study Investigators. Atherosclerosis Risk in Communities. Arterioscler Thromb Vasc Biol 17, 1171-1177.

37. El-Sohemy A, Baylin A, Kabagambe E, et al. (2002) Individual carotenoid concentrations in adipose tissue and plasma as biomarkers of dietary intake. Am J Clin Nutr 76, 172-179.

38. Jahns L, Johnson LK, Mayne ST, et al. (2014) Skin and plasma carotenoid response to a provided intervention diet high in vegetables and fruit: uptake and depletion kinetics. Am J Clin Nutr 100, 930-937.

39. Marshall JR (2003) Methodologic and statistical considerations regarding use of biomarkers of nutritional exposure in epidemiology. J Nutr 133, Suppl. 3, 881S-887S.

40. Rao AV (2002) Lycopene, tomatoes, and the prevention of coronary heart disease. Exp Biol Med (Maywood) 227, 908-913.

41. Fiedor J \& Burda K (2014) Potential role of carotenoids as antioxidants in human health and disease. Nutrients $\mathbf{6}$, 466-488.

42. Ciccone MM, Cortese F, Gesualdo M, et al. (2013) Dietary intake of carotenoids and their antioxidant and antiinflammatory effects in cardiovascular care. Mediators Inflamm 2013, 782137.

43. Coyne T, Ibiebele TI, Baade PD, et al. (2009) Metabolic syndrome and serum carotenoids: findings of a cross-sectional study in Queensland, Australia. Br J Nutr 102, 1668-1677.

44. Sugiura M, Nakamura M, Ogawa K, et al. (2008) Associations of serum carotenoid concentrations with the metabolic syndrome: interaction with smoking. Br J Nutr 100, 1297-1306.

45. Hozawa A, Jacobs DR Jr, Steffes MW, et al. (2007) Relationships of circulating carotenoid concentrations with several markers of inflammation, oxidative stress, and endothelial dysfunction: the Coronary Artery Risk Development in Young Adults (CARDIA)/Young Adult Longitudinal Trends in Antioxidants (YALTA) study. Clin Chem 53, 447-455. 